\title{
THE ALGEBRA OF THE FINITE FOURIER TRANSFORM AND CODING THEORY
}

\author{
BY \\ R. TOLIMIERI ${ }^{1}$
}

\begin{abstract}
The role of the finite Fourier transform in the theory of error correcting codes has been explored in a recent text by Richard Blahut. In this work we study how the finite Fourier transform relates to certain polynomial identities involving weight enumerator polynomials of linear codes. These include the generalized MacWilliams identities and theorems originally due to R. Gleason concerning polynomial algebras containing weight enumerator polynomials. The Heisenberg group model of the finite Fourier transform provides certain algebras of classical theta functions which will be applied to reprove Gleason's results.
\end{abstract}

I. Introduction. This work will place the theory of the finite Fourier transform at center stage in the study of several problems in coding theory, especially those involving the weight enumerator polynomial and its characterization. The emergence of finite Fourier transform theory as an explicit tool in coding theory can be found in R. Blahut's recently published text $[\mathbf{1 0}]$ Theory and practice of error control codes, where many important aspects of coding theory are viewed within the framework of the finite Fourier transform. That this should be the case should come as no surprise to coding theorists, who often apply the Hadamard transform to coding theory problems. The Blahut text formalizes this cohabitation of ideas and has been a major influence on this work.

In $\S I I$ we present the theory of the finite Fourier transform on a finite-dimensional vector space over finite fields. We then show how the MacWilliams identities for exact weight enumerator polynomials of linear codes follow (up to rather complicated, but standard, algebraic identifications) from the definition of the Fourier transform.

The second major influence on this work can be found in the work of N. Sloane [8]. There he applies theta function theory to the study of weight enumerator polynomials. In [1] L. Auslander and the author built an algebra from the finite Fourier transform on $n$ points, $n>0$, that was related to an algebra of theta functions.

We now introduce some notation and definitions that enable us to review the results in $[\mathbf{1}]$.

For an integer $m>0$, denote by $Z / m$ the integers $\bmod m$, and by $L_{m}$ the space of all complex-valued functions on $Z / m$, along with the inner product $\langle f, g\rangle$ on $L_{m}$

Received by the editors August 5, 1982 and, in revised form, February 3, 1984.

1980 Mathematics Subject Classification. Primary 22E25, 22E40, 94B05, 43A80.

${ }^{1}$ Partially supported by N.S.F. 
defined by

$$
\langle f, g\rangle=\sum_{j=0}^{m-1} f(j) \bar{g}(j),
$$

where bar denotes complex conjugate.

Let us now introduce two orthogonal bases of the $m$-dimensional vector space $L_{m}$ which we need in this work. For each $0 \leq j<m$ let $x_{j} \in L_{m}$ be defined by

$$
x_{j}(k) \equiv\left\{\begin{array}{ll}
1, & k=j \\
0, & k \neq j,
\end{array} \quad 0 \leq k<m\right.
$$

Direct calculation shows that

$$
\left\langle x_{j}, x_{k}\right\rangle \equiv\left\{\begin{array}{ll}
1, & j=k, \\
0, & j \neq k,
\end{array} \quad 0 \leq j, k<m,\right.
$$

and. hence, $x_{0}, x_{1}, \ldots, x_{m-1}$ is an orthonormal basis of $L_{m}$.

For each $0 \leq j<m$ define $\Psi_{j} \in L_{m}$ by

$$
\Psi_{j}(k) \equiv \exp (2 \pi i j k / m), \quad 0 \leq k<m .
$$

It is easy to see that

$$
\left\langle\Psi_{j}, \Psi_{k}\right\rangle=\left\{\begin{array}{ll}
m, & j=k, \\
0, & j \neq k,
\end{array} \quad 0<j, k<m,\right.
$$

and, hence, $\Psi_{0}, \Psi_{1}, \ldots, \Psi_{m-1}$ is an orthogonal basis of $L_{m}$.

The set

$$
\widehat{Z_{m}} \equiv\left\{\Psi_{j}: 0 \leq j<m\right\}
$$

is a group with respect to the multiplication

$$
\Psi_{j} \Psi_{k} \equiv \Psi_{j+k}, \quad 0 \leq j, k<m,
$$

where $j+k$ is taken $\bmod m$. We call $\widehat{Z_{m}}$ the dual, or character, group of $Z_{m}$.

The Fourier transform $F_{m}$ on $Z / m$ is the linear mapping of $L_{m}$ defined by setting

$$
F_{m}\left(x_{j}\right)=(1 / \sqrt{m}) \Psi_{j} . \quad 0 \leq j<m .
$$

Clearly. $F_{m}$ is a unitary operator on $L_{m}$ and, for any $f \in L_{m}$, we have

$$
F_{m}(f)(k)=\frac{1}{\sqrt{m}} \sum_{j=0}^{m-1} f(j) \exp \left(2 \pi i \frac{j k}{m}\right), \quad 0 \leq k<m .
$$

Consider

$$
\begin{array}{ll}
L=\sum_{m \geq 0} \oplus L_{m}, & L_{0} \equiv \mathbf{C}, \\
F=\sum_{m \geq 0} \bigoplus F_{m}, & F_{0} \equiv \text { identity mapping. }
\end{array}
$$

In $[\mathbf{1}]$ we proved that algebra structures can be defined on $L$ so that $F$ becomes an algebra homomorphism. One of these is isomorphic to the algebra of theta functions on $\mathbf{C}(a+i b), a, b \in Z$. This algebra will play a crucial role in our analysis of Gleason's theorems stated in §IV. 
The main coding theorems to be proved by our methods are the MacWilliams identities, Theorems A and B, in $\S I I$ and the Gleason theorems, Theorems C and $\mathrm{D}$, in $\S \mathrm{IV}$. The tools for the MacWilliams identities are contained in $\S \mathrm{II}$, while those for Gleason's results appear in §III. Original proofs of these results can be found in [4 and 3]. Other proofs of Gleason's results can be found in [5 and 9].

II. The Fourier transform and coding theory. Consider a prime $p$ and denote the field of integers $\bmod p$ by $Z / p$. Let $n \geq 1$ be an integer and denote $n$ dimensional vector space over $Z / p$ by $V_{n}$. Since, in the first part of this discussion, $n$ will be fixed, we set $V \equiv V_{n}$. A typical point $v \in V$ will be written $v=$ $\left(v_{1}, \ldots, v_{n}\right), v_{j} \in Z / p$.

A linear code in $V$ or a linear code of length $n$ over $Z / p$ is any subspace $C$ of $V$. The dimension of the linear code $C$ in $V$ is its dimension as a vector space over $Z / p$.

An inner product on $V$ will now be specified. The inner product of two elements $v, w \in V$, denoted by $\langle v, w\rangle$, is defined by

$$
\langle v, w\rangle=\sum_{j=1}^{n} v_{j} w_{j} .
$$

The dual $C^{\perp}$ of a linear code $C$ in $V$ is given by

$$
C^{\perp}=\{v \in V:\langle v, w\rangle=0 \text { for all } w \in C\} .
$$

It is easy to see that $C^{\perp}$ is also a linear code. We say that $C$ is self-dual whenever

$$
C^{\perp}=C
$$

Consider $V$ as an abelian group under addition. A character of $V$ is any mapping $\Psi: V \rightarrow \mathbf{C}^{x}$ satisfying

$$
\Psi(v+w)=\Psi(v) \cdot \Psi(w), \quad v, w \in V .
$$

The set of all characters of $V$, denoted $\hat{V}$, becomes a group under the product rule

$$
\left(\Psi \Psi^{\prime}\right)(v) \equiv \Psi(v) \Psi\left(v^{\prime}\right), \quad \Psi, \Psi^{\prime} \in \hat{V}, v \in V .
$$

The mapping $\Psi_{0}$, given by $\Psi_{0}(v) \equiv 1, v \in V$, is called the trivial character on $V$. The following formulas will be used without proof:

$$
\begin{gathered}
\sum_{v \in V} \Psi(v)=0 \quad \text { if } \Psi \text { is not the trivial character. } \\
\qquad \sum_{\Psi \in \hat{V}} \Psi(v)=0 \quad \text { if } v \neq 0 .
\end{gathered}
$$

Up to this point we have made no use of the special nature of $V$. Definitions (2.4) and (2.5), as well as formulas (2.6) and (2.7), carry over to any finite abelian group $A$. Although it is true that for any finite abelian $A$ we have $A$ and $\hat{A}$ isomorphic. we use the inner product (2.1) to define an isomorphism between $V$ and $\hat{V}$. This isomorphism is not canonically defined in terms of $V$ as an abelian group, but essentially depends upon our choice of inner product. 
For $v \in V$ we define the mapping $\Psi_{v}$ by the rule

$$
\Psi_{v}(w) \equiv \exp (2 \pi i(1 / p)\langle v, w\rangle), \quad v, w \in V,
$$

where we view an element of $Z / p$ as its representative $k$ in $Z$ such that $0 \leq k<p$. It is easy to verify that $\Psi_{v} \in \hat{V}$, and the mapping $v \mapsto \Psi_{v}, v \in V$, defines an isomorphism from $V$ onto $\hat{V}$.

Let $L^{2}(V)$ denote the set of all complex-valued functions on $V$ made into an inner product space by setting

$$
\langle f, g\rangle \equiv \sum_{v \in V} f(v) \bar{g}(v), \quad f, g \in L^{2}(V) .
$$

The Fourier transform of $V$, denoted $F_{V}$, is the mapping of $L^{2}(V)$ to itself defined by

$$
F_{V}(f)(w) \equiv p^{-n / 2} \sum_{v \in V} f(v) \Psi_{v}(w),
$$

where $w \in V$ and $f \in L^{2}(V)$. A straightforward verification shows that $F_{V}$ is a unitary operator of $L^{2}(V)$. To simplify notation we write $F$ for $F_{V}$.

The evaluation function of a subset $S$ of $V$, denoted $E_{S}$, is the function on $V$ given by the rule

$$
E_{S}(v) \equiv\left\{\begin{array}{ll}
1, & v \in S, \\
0, & v \notin S,
\end{array} \quad v \in V .\right.
$$

If $\{v\}$ denotes the set $v$, we let $E_{\{v\}}=E_{v}$. Clearly, the set of functions

$$
E_{v}, \quad v \in V
$$

determines an orthonormal basis of $L^{2}(V)$.

THEOREM 2.1. Let $C$ be a linear code in $V$. Then

$$
F\left(E_{C}\right)=o(C) p^{-n / 2} E_{C^{\perp}},
$$

where $F$ is the Fourier transform of $V$, and $o(C)$ equals the number of elements in C.

PROOF. By definition,

$$
F\left(E_{C}\right)(v)=p^{-n / 2} \sum_{w \in C} \Psi_{v}(w), \quad v \in V
$$

If $v \in C^{\perp}$ then $\Psi_{v}(w)=1$ for all $w \in C$ and we have

$$
F\left(E_{C}\right)(v)=o(C) p^{-n / 2} .
$$

If $v \notin C^{\perp}$ then $\Psi_{v}$, restricted to $C$ is a nontrivial character of the abelian group $C$ and, by (2.6), $\sum_{w \in C} \Psi_{v}(w)=0$, which completes the proof of the theorem.

Theorem 2.1 is a disguised version of the MacWilliams identity for the exact weight enumerator polynomial. To recognize (2.13) as a MacWilliams identity, certain well-known identifications will be recalled. 
Let $X \equiv L^{2}(Z / p)$, the space of all complex-valued functions on $Z / p$. We consider $X$ as a $p$-dimensional vector space over $\mathbf{C}$. We know that a basis for $X$ is given by the functions

$$
x_{0}, x_{1}, \ldots, x_{p-1}
$$

where

$$
x_{j}(k) \equiv\left\{\begin{array}{ll}
1, & k=j, \\
0, & k \neq j,
\end{array} \quad 0 \leq j, k<p\right.
$$

We let

$$
T_{n}(X)=\bigotimes_{1}^{n} X_{j}, \quad X_{j}=X, j=1, \ldots, n,
$$

denote the tensor product of $n$ copies of $X$. In the following discussion we set $T_{n} \equiv T_{n}(X)$.

Consider $v \in V$ and let $x^{v} \in T_{n}$ be defined by

$$
x^{v} \equiv \bigotimes_{j=1}^{n} x_{v_{j}}
$$

where $v=\left(v_{1}, \ldots, v_{n}\right)$ and $v_{j} \in Z / p$. We call $x^{v}$ a monomial of degree $n$. The set of all monomials of degree $n$,

$$
x^{v}, \quad v \in V,
$$

defines a basis of $T_{n}$. An arbitrary $f \in T_{n}$ can be written

$$
f \equiv f(x) \equiv \sum_{v \in V} a(v) x^{v}, \quad a(v) \in \mathbf{C},
$$

and will be called a homogeneous polynomial in the noncommuting variables (2.14) of degree $n$.

THEOREM 2.2. Let $\phi_{n}$ be the linear mapping $\phi \equiv \phi_{n}: T_{n} \rightarrow L^{2}(V)$ defined on the basis elements (2.17) by the rule

$$
\phi\left(x^{v}\right) \equiv E_{v}, \quad v \in V .
$$

Then $\phi$ is an isomorphism of $T_{n}$ onto $L^{2}(V)$.

Moreover, for $f \in T_{n}$ of the form

$$
f=\bigotimes_{j=1}^{n} f_{j}, \quad f_{j} \in X
$$

we have

$$
\phi(f)(v)=\prod_{j=1}^{n} f_{j}\left(v_{j}\right)
$$

where $v=\left(v_{1}, \ldots, v_{n}\right) \in V$.

ProQf. Since the set of functions $E_{v}, v \in V$, defines a basis of $L^{2}(V), \phi$ is trivially an isomorphism of $T_{n}$ onto $L^{2}(V)$. 
Observe that, by definition, $f_{j}$ is a function on $Z / p$. Formula (2.20) clearly holds whenever $f$ is of the form $f=x^{w}, w \in V$. In general, write

$$
f_{j}=\sum_{k=0}^{p-1} a_{j}(k) x_{k}, \quad a_{j}(k) \in \mathbf{C},
$$

and use the bilinearity of the tensor product to finish the proof of the theorem.

Consider the Fourier transform $F \equiv F_{v}$ of $V$. Since $\phi$ is a linear isomorphism,

$$
F^{\phi} \equiv \phi^{-1} \circ F \circ \phi
$$

is a linear isomorphism of $T_{n}$. The next theorem describes the action of $F^{\phi}$ on $T_{n}$. Recall that $F_{p}$ denotes the Fourier transform on $L_{p}$. For the sake of completeness we state and prove the following known result.

THEOREM 2.3. $F=\bigotimes_{1}^{n} F_{p}$.

ProOf. Consider $x^{v}, v \in V$. By definition

$$
\left(\bigotimes_{1}^{n} F_{p}\right)\left(x^{v}\right)=\bigotimes_{1}^{n}\left(F_{p}\left(x_{j}\right)\right)
$$

and. by $\S I$.

$$
F_{p}\left(x_{j}\right)=p^{-1 / 2} \sum_{k=0}^{p-1} \exp \left(2 \pi i \frac{1}{p} j k\right) x_{k}
$$

Thus.

Since $(\phi \circ F)\left(x^{v}\right)=F\left(E_{v}\right)$ and

$$
\begin{aligned}
\left(\phi \circ\left(\bigotimes_{j=1}^{n} F_{p}\right)\right)\left(x^{v}\right)(w) & =\prod_{j=1}^{n} F_{p}\left(x_{v_{j}}\right)(w) \\
& =p^{-n / 2} \prod_{j=1}^{n} \exp \left(2 \pi i \frac{1}{p} v_{j} w_{j}\right) \\
& =p^{-n / 2} \exp \left(2 \pi i \frac{1}{p}\langle v, w\rangle\right) .
\end{aligned}
$$

$$
F\left(E_{v}\right)(w)=p^{-n / 2} \exp (2 \pi i(1 / p)\langle v, w\rangle),
$$

the theorem follows.

Consider $f \in T_{n}$ and write

$$
f=\sum_{v \in V} a(v) x^{v}, \quad a(v) \in \mathbf{C} .
$$

We can use Theorem 2.3 to explicitly write $F^{\phi}(f)$. Let

$$
y_{j}=p^{-1 / 2} \sum_{e=0}^{p-1} \exp \left(2 \pi i \frac{1}{p} j k\right) x_{k}, \quad 0 \leq j<p .
$$

For $v \in V$ we write

$$
y^{\prime \prime}=y_{v_{1}} \otimes \bigotimes y_{v_{n}}, \quad v=\left(v_{1}, \ldots, v_{n}\right) .
$$

An immediate application of Theorem 2.3 gives the following result. 
THEOREM 2.4. $F^{\phi}(f)=\sum_{v \in V} a(v) y^{v}$.

We come now to the MacWilliams identities. The exact weight enumerator of a linear code $C$ on $V$, denoted $P_{C}$, is defined by

$$
P_{C} \equiv P_{C}(x) \equiv \sum_{v \in C} x^{v}
$$

By definition of $\phi$ we have

$$
\phi\left(P_{C}\right)=E_{C}
$$

and, hence, by Theorem 2.1,

$$
F^{\phi}\left(P_{C}\right)=o(C) p^{-n / 2} P_{C^{\perp}}
$$

The MacWilliams identity for exact enumerator polynomials immediately follows from Theorem 2.4 and is given by the next theorem.

THEOREM A. If $P_{C}$ is the exact enumerator polynomial of a linear code $C$ on $V$, then

$$
P_{C}(y)=o(C) p^{-n / 2} P_{C^{\perp}}(x)
$$

where $y$ is given by (2.22).

The exact weight enumerator polynomial $P_{C}$ of the linear code $C$ specifies the code uniquely but, involving as it does noncommuting variables, it is difficult to handle. A related polynomial, the complete weight enumerator polynomial, contains less information, but still reflects essential features of the code and has the advantage of involving commuting variables only.

We begin by a direct definition of the complete weight enumerator polynomial of a code. Consider a linear code $C$ in $V \equiv V_{n}$ over $Z / p$. For any element $v=$ $\left(v_{1}, \ldots, v_{n}\right) \in V$ and any $k, 0 \leq k<p$, we set $w_{k}(v)$ equal to the number of $v_{j}, 1 \leq$ $j \leq n$, satisfying $v_{j} \equiv k \bmod p$. The complete weight enumerator polynomial, $W \equiv$ $W_{C}$, of $C$ is defined by setting

$$
W_{C}(x)=\sum_{v \in C} x^{w(v)}
$$

where $x^{w(v)}=x_{0}^{w_{0}(v)} \cdots x_{p-1}^{w_{p-1}(v)}$.

We observe that $W_{C}(x)$ is a homogeneous polynomial of degree $n$ in the $p$ commuting variables $x_{0}, \ldots, x_{p-1}$. Also, if $C$ is a self-dual code, then $n$ must have been even.

Let us now define the complete weight enumerator polynomial in terms of the exact weight enumerator polynomial. Consider the vector space defined by

$$
T \equiv \sum_{m \geq 0} \bigoplus T_{m}
$$

We briefly review the definition of the tensor algebra over $X$ and how it is isomorphic to the polynomial algebra over $\mathbf{C}$ in $p$ noncommuting variables.

For $m \geq 1$ the monomials of degree $m, x^{v}, v \in V_{m}$, form a basis of $T_{m}$. Take $v \in V_{l}$ and $u \in V_{m}, l, m \geq 1$, and define the product $x^{v} \cdot x^{u}$ by the rule

$$
x^{v} \cdot x^{u}=x^{w},
$$


where $w=(v, u) \in V_{l+m}$. Extending (2.27) to a product on the vector space

$$
\sum_{m \geq 1} \bigoplus T_{m}
$$

by linearity and the distributive law determines a unique algebra structure on (2.28). Since $T_{(0)} \equiv \mathbf{C}$, this algebra product extends to an algebra product on $T$.

Suppose the algebra $T$ is given as above. Consider the ideal $I$ of $T$ generated by the relations

$$
x_{j} x_{k}=x_{k} x_{j}, \quad 0 \leq j, k<p .
$$

$I$ is homogeneous relative to the decomposition of $T$, given by $(2.26)$, in the sense that

$$
I=\sum_{m \geq 0} \bigoplus I_{m}
$$

where $I_{m}=I \cap T_{m}$. Note $I_{0}=I_{1}=(0)$.

It follows that the quotient algebra $T / I$ is isomorphic to $\mathbf{C}\left[x_{0}, \ldots, x_{p-1}\right]$, the polynomial algebra over $\mathbf{C}$ in the commuting variables $x_{0}, \ldots, x_{p-1}$, and as vector spaces.

$$
T / I=\sum_{m \geq 0} \bigoplus T_{m} / I_{m}
$$

We call $T / I$ the full symmetric algebra of $X$.

Consider the natural map $\eta$ of $T_{n}$ onto $T_{n} / I_{n}$. Let $P_{C}$ be the exact enumerator polynomial of the linear code $C$ in $V=V_{n}$. Then $P_{C} \in T_{n}$. The complete weight cmumerator polynomial of $C$, denoted $W_{C}$, is defined by setting

$$
W_{C}=\eta\left(P_{C}\right) \text {. }
$$

The MacWilliams identity for the complete weight enumerator polynomial of a lincar code immediately follows from Theorem A.

THEOREM B. Let $C$ be a linear code and $W_{C}$ its complete weight enumerator polynomial. Then.

$$
W_{C}(y)=o(C) p^{-n / 2} W_{C}(x),
$$

where $y$ is given by $(2.22)$.

III. The algebra of the Fourier transform. A prime $p$ is fixed for the remainder of this section. We also set $L_{m} \equiv L(Z / m), m \geq 1$. The Fourier transform of $L_{m}$ is denoted by $F_{m}$.

As a prelude to Gleason's results, we now relate the Fourier transform to the algebras introduced in the previous section.

Take a fixed integer $n \geq 1$. Let $F$ denote the Fourier transform of $V \equiv V_{n}$, and let $\phi$ be the linear isomorphism from $T_{n}$ onto $L^{2}(V)$ defined in Theorem 2.2. Consider the lincar isomorphism $F^{\phi} \equiv \phi^{-1} \circ F \circ \phi$ of $T_{n}$. A typical element in $I_{n}$ is the sum of elements of the form $f(x)\left(x_{j} x_{k}-x_{k} x_{j}\right) g(x)$, where $f, g \in T$, of proper degrees. Applying $F^{\infty}$ to this element. by Theorem 2.4, implies $f(y)\left(y_{j} y_{k}-y_{k} y_{j}\right) g(y)$, where $y$ is given by (2.22). Expanding this, we easily see that this latter element is in $I_{n}$. Thus.

$$
F^{\phi}\left(I_{n}\right) \subset I_{n}
$$


and $F$ induces a linear homomorphism of the vector space $T_{n} / I_{n}$. We denote the induced linear isomorphism of $T_{n} / I_{n}$ by $F(n)$.

The Fourier transform of $T / I$, denoted by $F_{S}$, is defined by

$$
F_{S}=\sum_{n \geq 0} \bigoplus F(n)
$$

where $F(0)$ is the identity mapping. The definition of tensor products immediately implies the next result.

THEOREM 3.1. $F_{S}$ is an algebra homomorphism of $S=T / I$ and is uniquely determined by its action on the free generators $x_{0}, \ldots, x_{p-1}$ of $S$. The rule for this action is

$$
F_{S} x_{j}=\frac{1}{\sqrt{p}} \sum_{e=0}^{p-1} \exp \left(2 \pi i \frac{1}{p} j k\right) x_{k}, \quad 0 \leq j, k<p .
$$

We introduce at this time two other important algebra homomorphisms of $S$. Since $x_{0}, \ldots, x_{p-1}$ freely generate $S$, it suffices to define the homomorphism action on these generators.

We define the algebra homomorphisms $R_{S}$ and $U_{S}$ of $S$ by the formulas

$$
\begin{aligned}
& R_{S} x_{j} \equiv x_{j+1}, \\
& U_{S} x_{j} \equiv \exp (2 \pi i j / p) x_{j},
\end{aligned}
$$

where $0 \leq j<p$. If $j=p-1$ in (3.4), then $j+1=p$ is set equal to 0 .

THEOREM 3.2. $F_{S} R_{S} F_{S}^{-1}=U_{S}$.

Proof. By Definitions (3.3) and (3.4) we have

$$
F_{S} R_{S} x_{j}=\frac{1}{\sqrt{p}} \sum_{k=0}^{p-1} \exp \left(2 \pi i \frac{1}{p}(j+1) k\right) x_{k},
$$

and by Definition (3.5),

$$
\begin{aligned}
U_{S} F_{S} x_{j} & =\frac{1}{\sqrt{p}} \sum_{k=0}^{p-1} \exp \left(2 \pi i \frac{1}{p} j k\right) U_{S} x_{k} \\
& =\frac{1}{\sqrt{p}} \sum_{k=0}^{p-1} \exp \left(2 \pi i \frac{1}{p} j k\right) \exp \left(2 \pi i \frac{1}{p} k\right) x_{k} \\
& =F_{S} R_{S} x_{j},
\end{aligned}
$$

which proves the theorem.

The algebra $T / I \equiv S$ is too 'big' for our purposes, in a sense described below. We now define other algebras upon which a Fourier transform acts. The first of these was introduced in $[\mathbf{1}]$, and we briefly outline the results needed.

Consider the vector space

$$
L \equiv \sum_{m \geq 0} \bigoplus L_{m}
$$


and the linear mapping of $L$,

$$
F_{L} \equiv \sum_{m \geq 0} \bigoplus F_{m}
$$

We call $F_{L}$ the Fourier transform of $L$.

The following two theorems can be found in $[\mathbf{1}]$.

Algebra THEOREM. Up to isomorphism, $L$ admits exactly three algebra structures, denoted by $L^{\alpha}, x=1,2,3$, satisfying:

(1) if $f \in L_{m}$ and $g \in L_{n}$, then $f g \in L_{m+n}$;

(2) $L^{\alpha}$ has no zero divisors; and

(3) $F_{L}$ is an algebra homomorphism of $L^{\alpha}$.

Consider the action of $F_{L}$ on $L_{j}, j=1,2,3$. A direct computation shows that, up to constant multiple, there exist unique elements

$$
z_{j} \in L_{j}, \quad j=1,2,3
$$

satisfying

$$
F_{L}\left(z_{1}\right)=z_{1}, \quad F_{L}\left(z_{2}\right)=-z_{2}, \quad F_{L}\left(z_{3}\right)=i z_{3} .
$$

REPRESENTATION THEOREM. The algebra $L^{\alpha}$ is generated by the elements $z_{1}, z_{2}$ and $z_{3}$. The homomorphism of algebras $\pi^{\alpha}: \mathbf{C}\left[Z_{1}, Z_{2}^{2}, Z_{3}^{3}\right] \rightarrow L^{\alpha}$, defined by requiring $\pi^{\alpha}\left(Z_{j}^{j}\right) \equiv z_{j}, j=1,2,3$, induces an isomorphism $\mathbf{C}\left[Z_{1}, Z_{2}^{2}, Z_{3}^{3}\right] / p_{\alpha} \cong L^{\alpha}$, where we can take

$$
p_{1}=Z_{3}^{6}+Z_{2}^{6}, \quad p_{2}=Z_{3}^{6}+Z_{1}^{4} Z_{2}^{2}+Z_{2}^{6}, \quad p_{3}=Z_{3}^{6}+Z_{1}^{4} Z_{2}^{2} .
$$

In $L^{\alpha}$ the set of monomials given by

$$
z^{a}=z_{1}^{a_{1}} z_{2}^{a_{2}} z_{3}^{a_{3}}, \quad a=\left(z_{1}, a_{2}, a_{3}\right), a_{j} \geq 0,
$$

spans the vector space $L$. Thus, $z^{a}$, where $m=a_{1}+a_{2}+a_{3}$, spans the subspace $L_{m}, m \geq 1$, of $L^{\alpha}$. Moreover, since $F_{L}$ is an algebra homomorphism of $L$, we have

$$
F_{L}\left(z^{a}\right)=(-1)^{a_{2} \cdot a_{3}} z^{a} \text {. }
$$

For each $m \geq 1$, a basis $B(m)$ of $L_{m}$ can be defined inductively, consisting of monomials $z^{a}, m=a_{1}+a_{2}+a_{3}$. Let $B(1) \equiv\left\{z_{1}\right\}$. Suppose a basis $B(m-1)$ of $L_{m-1}$ has been constructed. We define $B(m)$ to be the set of functions of $L_{m}$ given by

$$
B(m)= \begin{cases}\left\{z_{1} B(m-1), z_{2}^{m / 2}\right\}, & m \text { even } \\ \left\{z_{1} B(m-1), z^{(m-3) / 2} \cdot z_{3}\right\}, & m \text { odd }\end{cases}
$$

It follows from the discussion in $[\mathbf{1}]$ that $B(m)$ is a basis of $L_{m}$.

Consider $L_{p}$ as a subspace of the algebra $L^{\alpha}$. The basis elements $x_{0}, \ldots, x_{p-1}$ of $L_{p}$ need not be free on $L^{\alpha}$, in the sense that some polynomial in $x_{0}, \ldots, x_{p-1}, F(x)$, given by $f(x)=\sum a(v) x^{v}$, can be 0 in $L^{\alpha}$ with some $a(v) \neq 0$. In fact, some linear combination must already exist between the monomials $x_{j} x_{k}, 0 \leq j, k<p$, and there are $p(p+1) / 2$ such monomials which all lie in $L_{2 p}$, a vector space of dimension $2 p$.

Denote by $A^{\alpha}(p)$ the subalgebra of $L$ generated by $L_{p}$. Then $x_{0}, \ldots, x_{p-1}$ generate $A^{\alpha}(p)$. The next result is an immediate consequence of $F_{L}$ being an algebra homomorphism. 
THEOREM 3.3. The Fourier transform $F_{L}$ maps $A^{\alpha}(p)$ onto itself and is uniquely determined by its action on the generators $x_{0}, \ldots, x_{p-1}$. This action is given by (3.3).

Since the algebra $S \equiv T / I$ is freely generated by $x_{0}, \ldots, x_{p-1}$, we can define an algebra homomorphism $\Psi^{\alpha}$ of $S$ onto $A^{\alpha}(p)$ by requiring $\Psi^{\alpha}\left(x_{j}\right) \equiv x_{j}, 0 \leq j<p$. It follows automatically from Theorems 3.1 and 3.2 that the next result holds.

THEOREM 3.4. $F_{L} \Psi^{\alpha}=\Psi^{\alpha} F_{S}$.

Observe that Theorem 3.4 implies

$$
F_{S}\left(\operatorname{ker} \Psi^{\alpha}\right) \subset \operatorname{ker} \Psi^{\alpha}
$$

We would like the algebra homomorphisms $R_{S}$ and $U_{S}$ of $S$ to induce, under $\Psi^{\alpha}$, algebra homomorphisms of $A^{\alpha}(p)$. Necessary and sufficient conditions for this to occur are

$$
\begin{aligned}
& R_{S}\left(\operatorname{ker} \Psi^{\alpha}\right) \subset \operatorname{ker} \Psi^{\alpha}, \\
& U_{S}\left(\operatorname{ker} \Psi^{\alpha}\right) \subset \operatorname{ker} \Psi^{\alpha} .
\end{aligned}
$$

From the discussion in $[\mathbf{1}]$ we can prove that, for at least one $\alpha=1,2,3$, these conditions hold. Even more can be said, which we develop below.

For an integer $r \geq 1$ we define the subalgebra $L^{\alpha}[r]$ of $L^{\alpha}$ by

$$
L^{\alpha}[r] \equiv \sum_{m \geq 1} \bigoplus L_{r m}
$$

Observe that $A^{\alpha}(p) \subset L^{\alpha}[p]$.

On $L^{\alpha}[p]$ we define the linear homomorphisms $R$ and $U$ as follows. For each $m \geq 1$ we define the linear homomorphisms $R_{m}$ and $U_{m}$ of $L_{p m}$ by

$$
\begin{gathered}
\left(R_{m} f\right)(k) \equiv f(k-m), \\
\left(U_{m} f\right)(k) \equiv \exp (2 \pi i k / p) f(k),
\end{gathered}
$$

where $f \in L_{p m}$ and $0 \leq k<p m$. Define $R$ and $U$ by

$$
\begin{aligned}
& R \equiv \sum_{m \geq 1} \bigoplus R_{m}, \\
& U \equiv \sum_{m \geq 1} \bigoplus U_{m} .
\end{aligned}
$$

The analogue of Theorem 3.2 holds on $L^{\alpha}[p]$. We state it without proof.

THEOREM 3.5. $F_{L} R F_{L}^{-1}=U$ on $L^{\alpha}[p]$.

One of the algebras $L^{\alpha}$ is classical, in that it can be realized as the algebra of theta functions of period $i$ relative to the standard lattice $Z+i Z$ in $\mathbf{C}$. The proof can be found in $[\mathbf{1}]$. Let $L^{*}$ denote this algebra. The proof of the following result can also be found in $[\mathbf{1}]$. We adopt the notational rule that we replace $\alpha$ by ${ }^{*}$ when referring to concepts based on $L^{*}$. 
HOMOMORPHISM THEOREM. $R$ and $U$ are algebra homomorphisms of $L^{*}[p]$ whose actions on $x_{0}, \ldots, x_{p-1}$ are given by (3.4) and (3.5), respectively.

Observe that since $F_{L}$ restricts to an algebra homomorphism of $L^{*}[p]$, by Theorem 3.5, $U$ is an algebra homomorphism if and only if $R$ is.

The following result immediately follows from the homomorphism theorem.

THEOREM 3.6. $A^{*}(p)$ is $R$ - and U-invariant. Moreover, we have

(1) $R \Psi^{*}=\Psi^{*} R_{S}$,

(2) $U \Psi^{*}=\Psi^{*} U_{S}$,

where $\Psi^{*}$ is the algebra homomorphism of $S$ onto the subalgebra $A^{*}(p)$ of $L^{*}[p]$.

Consider the subalgebra $L^{*}\left[p^{2}\right]$ of $L^{*}[p]$. Clearly $F_{L}$ restricts to an algebra homomorphism of $L^{*}\left[p^{2}\right]$. Also by the Homomorphism Theorem, $R$ and $U$ restrict to algebra homomorphisms of $L^{*}\left[p^{2}\right]$. Let

$$
\Theta \equiv\left\{f \in L^{*}\left[p^{2}\right]: R(f)=U(f)=f\right\} .
$$

THEOREM 3.7. $\Theta$ is a subalgebra of $L^{*}$ and

$$
F_{L}(\Theta)=\Theta .
$$

PROOF. Since $R$ and $U$ are algebra homomorphisms, $\Theta$ is a subalgebra. Theorem 3.5 implies (3.19).

$\Theta$ plays a fundamental role in our approach to Gleason's theorems. Consider the subspace $\Theta_{m}$ of $L_{p^{2} m}$. defined by

$$
\Theta_{m} \equiv \Theta \cap L_{p^{2} m}
$$

Since $L_{p^{2} m}$ is both $R$ - and $U$-invariant, it is easy to see that

$$
\Theta=\sum_{m \geq 0} \bigoplus \Theta_{m}
$$

$\Theta$ satisfies the following properties:

(3.22) (1) $\Theta$ has no zero divisors.

(2) If $f \in \Theta_{m}$ and $g \in \Theta_{m}$, then $f \cdot g \in \Theta_{m+n}$. By Theorem 3.7 we also have (3.23) (3) $\Theta$ is invariant under $F_{L}$.

Hence the restriction of $F_{L}$ to $\Theta$ is an algebra homomorphism.

Thus. $\Theta$ has properties similar to those satisfying the Algebra Theorem. The following important theorem implies it is, in fact, the algebra $L^{*}$ up to isomorphism. Thus. $L^{*}$ contains, as a proper subalgebra, an isomorphic copy of itself.

THEOREM 3.8. There exists an algebra isomorphism $D$ of $\Theta$ onto $L^{*}$ such that

(1) $D\left(\Theta_{m}\right) \subset L_{m}$.

(2) $D F_{L}=F_{L} D$ on $\Theta$.

Once we have the identification of $L^{*}$ with the theta function algebra, an easy proof would be available. However, we outline a proof without resorting to this identification.

We begin by describing the functions $f \in \Theta_{m}$. Since $U f=f$ we have

$$
f(k)=\exp (2 \pi i k / p) f(k), \quad 0 \leq k<m p^{2},
$$


and, hence, $f$ is "decimated"; i.e., $f(k)=0$ unless

$$
k=p j, \quad 0 \leq j<m p,
$$

in $Z / p^{2} m$. The condition $R f=f$ implies $f$ satisfies the periodicity property

$$
f(k)=f(k+m p), \quad 0 \leq k<p^{2} m .
$$

It follows that $f$ is completely determined by its values on the $m$ points

$$
0, p, z p, \ldots,(m-1) p .
$$

Conversely, if $g \in L_{m}$ we can define a function $f \in \Theta_{m}$ by setting $f(j p) \equiv g(j)$, $0 \leq j<m$, and requiring

$$
\begin{aligned}
f(k+m p) & \equiv f(k), \quad 0 \leq k<m p^{2}, \\
f(k) & \equiv 0, \quad p / k .
\end{aligned}
$$

This motivates the following definition. For $f \in \Theta_{m}$ define the function $D_{m}(f) \in$ $L_{m}$ by the rule

$$
D_{m}(f)(j) \equiv f(j p), \quad 0 \leq j<m .
$$

We have shown that $D_{m}$ is a linear isomorphism from $H_{m}$ onto $L_{m}$. Thus, setting

$$
D \equiv \sum_{m \geq 1} \bigoplus D_{m}
$$

we have that $D$ is a linear isomorphism from $\Theta$ onto $L$.

The Fourier transform $F_{L}$ acts on $L$ and, by Theorem 3.7, maps $\Theta$ onto itself. We will now see how $F_{L}$ acts relative to $D$.

Consider $f \in \Theta_{m}$. Since $F_{L}(f) \in \Theta_{m}$, it is determined by its values on the $m$ points (3.26). For $0 \leq j<m$ we have, using (3.24),

$$
F_{L}(f)(p j)=\frac{1}{p \sqrt{m}} \sum_{k=0}^{p m-1} f(k p) \exp \left(2 \pi i \frac{k j}{m}\right) .
$$

Write $k=k^{\prime}+k^{\prime \prime} m, 0 \leq k^{\prime}<m, 0 \leq k^{\prime \prime}<p$, and substitute in (3.29) to obtain

$$
\left(F_{L} f\right)(p j)=\frac{1}{\sqrt{m}} \sum_{k^{\prime}=0}^{m-1} f\left(k^{\prime} p\right) \exp \left(2 \pi i \frac{k^{\prime} j}{m}\right) \text {. }
$$

This implies

$$
D_{m}\left(F_{L}(f)\right)=F_{m}\left(D_{m}(f)\right)
$$

Therefore,

$$
D \circ F_{L}=F_{L} \circ D
$$

on $\Theta$.

It remains to show that $D$ is an algebra isomorphism of $\Theta$ onto $L^{*}$. There is a unique algebra structure on $L$, denoted $L \#$, such that $D$ is an algebra isomorphism of $\Theta$ onto $L \#$. But conditions (3.22) and (3.23), along with the preceding discussion, imply $L \#$ satisfies the conditions of the Algebra Theorem. Thus, $L \# \cong L^{\alpha}$ for some $\alpha=1,2,3$. Since $\Theta$ is a subalgebra of $L^{*}$, it follows from the Representation Theorem that $L \# \cong L^{*}$. This completes the proof of Theorem 3.8. 
The subalgebra $\Theta$ of $L^{*}\left[p^{2}\right] \subseteq L^{*}$ and certain of its subalgebras will play important roles in the study of weight enumerator polynomials. We introduce these subalgebras in the following discussion.

By Theorem 3.8 we know there exist uniquely determined, up to constant multiple, elements $w_{1} \in \Theta_{1}, w_{2} \in \Theta_{2}, w_{3} \in \Theta_{3}$ such that

$$
F_{L}\left(w_{1}\right)=w_{1}, \quad F_{L}\left(w_{2}\right)=-w_{2}, \quad F_{L}\left(w_{3}\right)=i w_{3},
$$

and $\Theta$ is generated as an algebra by $w_{1}, w_{2}, w_{3}$.

Moreover, we can choose a basis of $\Theta_{m}$ from the monomials $w^{a}=w_{1}^{a} w_{2}^{a} w_{3}^{a}, a=$ $\left(a_{1}, a_{2}, a_{3}\right)$, where $m=a_{1}+2 a_{2}+3 a_{3}$. Observe that the basis monomials always have $a_{3}=0$ or $a_{3}=1$. It follows from (3.11) that $w^{a}$ is an eigenvector of $F_{L}$ of eigenvalue \pm 1 if $a_{3}=0$; in addition, if $a_{2}$ is even, the eigenvalue is \pm 1 .

Denote by $\Theta^{\prime}$ the subalgebra of all $f \in H$ such that

$$
F_{L}(f)=f \text {. }
$$

Since each subspace $\Theta_{m}$ is $F_{L}$-invariant, we can write

$$
\Theta^{\prime}=\sum_{m \geq 0}\left(H_{m} \cap \Theta^{\prime}\right) .
$$

A basis of $\Theta_{m} \cap \Theta^{\prime}$ is given by the monomials

$$
w_{1}^{a_{1}} w_{2}^{2 a_{2}}, \quad a_{1}, a_{2} \in Z,
$$

where $a_{1}+4 a_{2}=m$. We also have, by the Representation Theorem, that $w_{1}, w_{2}$ freely generate. This proves

THEOREM 3.9. $\Theta^{\prime}=\mathbf{C}\left[w_{1}, w_{2}^{2}\right]$, where $w_{1}, w_{2}^{2}$ freely generate.

We need a slightly more general result.

THEOREM 3.10. Choose $y_{1} \in \Theta_{1}$ and $y_{2} \in \Theta_{4} \cap \Theta^{\prime}$ such that $y_{1}^{4}$ and $y_{2}$ are linearly independent. Then $\Theta^{\prime}=\mathbf{C}\left[y_{1}, y_{2}\right]$, where $y_{1}, y_{2}$ are free.

ProOF. $\Theta_{1}$ is one dimensional. Without loss of generality we can assume $y_{1}=w_{1}$. By (3.32) $w_{1}^{4}, w_{2}^{2}$ define a basis of $\Theta_{4} \cap \Theta^{\prime}$ and we can write $w_{2}^{2}=$ $a y_{1}^{4}+b y_{2}, b \neq 0$. Replacing $w_{2}^{2}$ by $a w_{1}^{2}+b y_{2}$ in (3.32), we get a polynomial in $w_{1}, y_{2}$. This implies $w_{1}, y_{2}$ generate $\Theta^{\prime}$.

To prove $w_{1}, y_{2}$ freely generate, we write $y_{2}=b^{-1}\left(w_{2}^{2}-a w_{1}^{4}\right)$ and argue as in the Appendix.

Two subalgebras of $\Theta^{\prime}$, defined by

$$
\begin{aligned}
& \Theta^{\prime \prime}=\left(\sum_{m \geq 0} \bigoplus \Theta_{2 m}\right) \cap \Theta^{\prime}, \\
& \Theta^{\prime \prime \prime}=\left(\sum_{m \geq 0} \bigoplus \Theta_{4 m}\right) \cap \Theta^{\prime},
\end{aligned}
$$

play an important role in $\S \mathrm{IV}$.

Arguing as in the preceding theorem, we have the following two results. 
THEOREM 3.11. If $y_{1} \in \Theta_{2} \cap \Theta^{\prime}$ and $y_{2} \in \Theta_{4} \cap \Theta^{\prime}$ satisfy the property that $y_{1}^{2}, y_{2}$ are linearly independent, then $y_{1}, y_{2}$ freely generate and $\Theta^{\prime \prime}=\mathbf{C}\left[y_{1}, y_{2}\right]$

THEOREM 3.12. Choose $y_{1}, y_{2} \in \Theta_{4} \cap \Theta^{\prime}$ such that they are linearly independent. Then $y_{1}, y_{2}$ freely generate $\Theta^{\prime \prime \prime}$.

For the rest of this section, $C$ denotes a self-dual code of length $n$ over $Z / p$ containing $1_{n}=(1,1, \ldots, 1)$. The existence of a self-dual code on the vector space $V \equiv V_{n}$ implies $n$ is even because, as is easy to show, as vector spaces $C \cong V / C^{\perp} \cong$ $V / C$ and $n=2 \operatorname{dim} C$. The condition $1_{n} \in C$ is equally important as a constraint on $n$ The inner product $\left\langle 1_{n}, 1_{n}\right\rangle=n$ must vanish $\bmod p$ and, hence, $p$ divides $n$. In particular, if $p$ is odd, then $2 p$ divides $n$.

Let $W=W_{C}$ be the complete weight enumerator polynomial of $C$. In general, $W$ is a homogeneus polynomial of degree $n$ in the commuting variables $x_{0}, \ldots, x_{p-1}$. This means that each monomial on $W$ has degree $n$ divisible by 2 and $p$.

The condition $1_{n} \in C$ further restrains the form of $W$. If $a=\left(a_{0}, \ldots, a_{p-1}\right)$ is a $p$-tuple of integers with $n=\sum_{j=0}^{p-1} a_{j}$, then the coefficient of the monomial $x^{a} \equiv x_{0}^{a_{0}} \cdots x_{p-1}^{a_{p-1}}$ in $W$ is equal to the number of code words $v \in C$ having, for each $j, a \leq j<p$, exactly $a_{j}$ components equal to $j$. Let $S$ denote the subset of such elements in $C$. Then $1_{n}+S$ is the subset of $C$ consisting of all code words $v \in C$ having, for each $j, 0 \leq j<p$, exactly $a_{j}$ components equal to $j+1 \bmod p$. Thus, the coefficient of $x^{a}$ on $W$ equals the coefficient of the monomial $x_{0}^{a_{p-1}} x_{1}^{a_{0}} \cdots x_{p-1}^{a_{p-2}}$ in $W$. This implies $W$ is invariant under the action of the shift $R_{S}$. We summarize in the following theorem what we have just shown about $W$.

THEOREM 3.13. Let $W \equiv W_{C}$ be the complete weight enumerator polynomial of the self-dual code $C$ in $V_{n}$ containing $1_{n}=(1, \ldots, 1)$. Then $W$ is a homogeneous polynomial of degree $n$ satisfying:

(1) 2 and $p$ divide $n$;

(2) $W=R_{S}(W)$;

(3) $W=F_{S}(W)$.

We note condition (3) is the MacWilliams identity, and (2) and (3) imply, by Theorem 3.2, that $W=U_{S}(W)$.

Certain subalgebras $A^{\prime}, A^{\prime \prime}, A^{\prime \prime \prime}$ of $S$ will now be introduced.

Let $S[p]$ be the subalgebra of $S$ defined by

$$
S[p]=\sum_{m \geq 0} \bigoplus T_{p m} / I_{p m}
$$

and consider the subalgebra $A^{\prime}$ of $S[p]$ defined as the set of all $f S[p]$ satisfying

(1) $R_{S}(f)=U_{S}(f)=f$, and

(2) $F_{S}(f)=f$.

Consider the algebra homomorphism $\Psi^{*}: S \rightarrow L^{*}[p]$.

THEOREM 3.14. $\Psi^{*}$ maps $A^{\prime}$ onto $\Theta^{\prime}$.

PROOF. Theorems 3.4 and 3.5 , along with the definition of $A^{\prime}$, imply

$$
\Psi^{*}(f)=U\left(\Psi^{*}(f)\right)=R\left(\Psi^{*}(f)\right)=F_{L}\left(\Psi^{*}(f)\right)
$$

whenever $f \in A^{\prime}$. We are done if we can show $\Psi^{*}(f) \in L^{*}\left[p^{2}\right]$ for all $f \in A^{\prime}$. It suffices to show that if $f \in A^{\prime}$ is a monomial given by $f=x^{v}$, where $q=\sum_{j=0}^{p-1} v_{j}$ 
is divisible by $p$, then $\Psi^{*}(f) \in L^{*}\left[p^{2}\right]$. Since $\Psi^{*}(f)=x^{v}$, where the product $x^{v}$ is taken in $L^{*}$, the theorem follows from observing that $\Psi^{*}(f) \in L_{p q} \subset L^{*}\left[p^{2}\right]$.

Consider the subalgebras

$$
S[2 p] \equiv \sum_{m \geq 0} \bigoplus T_{2 p m} / I_{2 p m}, \quad S[4 p] \equiv \sum_{m \geq 0} \bigoplus T_{4 p m} / I_{4 p m},
$$

$A^{\prime \prime} \subset S[2 p]$, and $A^{\prime \prime \prime} \subset S[4 p]$ such that

(1) $2 p$ divides $n$,

(2) $W=R_{S}(W)$.

The following theorem has a proof analogous to that of Theorem 3.14.

THEOREM 3.15. $\Psi^{*}$ maps $A^{\prime \prime}$ onto $\Theta^{\prime \prime}$ and $A^{\prime \prime \prime}$ into $\Theta^{\prime \prime \prime}$.

Gleason's theorems are related to the structure of the algebras $A^{\prime}, A^{\prime \prime}, A^{\prime \prime \prime}$ in cases $p=2$ or $p=3$. We present these results in the next section.

IV. Gleason's theorems. Take $p=2$ and consider the algebra homomorphism $\Psi^{*}: S \rightarrow L^{*}$. Arguing, as in the Appendix, it is easy to show that the elements $x_{0}, x_{1} \in L_{2} \subset L^{*}$ generate a free subalgebra of $L^{*}$. This implies $\Psi^{*}$ is a monomorphism. We note that 2 is the only prime for which we will be so lucky.

THEOREM C. The algebra homomorphism $\Psi^{*}$ maps $A^{\prime}$ isomorphically onto $\Theta^{\prime}$. If $f, g \in S$ are defined by

$$
f=x_{0}^{2}+x_{1}^{2}, \quad g=x_{0}^{2} x_{1}^{2}\left(x_{0}^{2}-x_{1}^{2}\right)^{2},
$$

then $f, g \in A^{\prime}$ freely generate and $A^{\prime}=\mathbf{C}[f, g]$.

Proof. The above discussion implies $\Psi^{*}$ is an algebra monomorphism of $A^{\prime}$ into $\Theta^{\prime}$. We want to prove $\Psi^{*}$ maps $A^{\prime}$ onto $\Theta^{\prime}$. At the same time we specify free generators of $A^{\prime}$. Since $F_{S}$ maps

$$
F_{S}\left(x_{0}\right)=\frac{1}{\sqrt{2}}\left(x_{0}+x_{1}\right) \quad \text { and } \quad F_{S}\left(x_{1}\right)=\frac{1}{\sqrt{2}}\left(x_{0}-x_{1}\right)
$$

we have

$$
F_{S}\left(x_{0}^{2}+x_{1}^{2}\right)=x_{0}^{2}+x_{1}^{2}, \quad F_{S}\left(x_{0} x_{1}\left(x_{0}^{2}-x_{1}^{2}\right)\right)=x_{0} x_{1}\left(x_{0}^{2}-x_{1}^{2}\right) .
$$

It follows that $f, g \in A^{\prime}$.

Note. $x_{0} x_{1}\left(x_{0}^{2}-x_{1}^{2}\right)$ is not $R_{S}$-invariant and, hence, does not belong to $A^{\prime}$.

Let $y_{1} \equiv \Psi^{*}(f)$ and $y_{2} \equiv \Psi^{*}(g)$. Since $f^{4}, g$ are linearly independent and $\Psi^{*}$ is an isomorphism, $y_{1}, y_{2}$ satisfy the hypothesis of Theorem 3.10. This implies they freely generate $\Theta^{\prime}$, and $f, g$ freely generate $A^{\prime}$, which completes the proof of the theorem.

Since $W \equiv W_{C} \in A^{\prime}$, whenever $C$ is a self-dual code over $Z / 2$, we have proved Gleason's theorem for the case $p=2$.

GLEASON'S THEOREM FOR $p=2$. Let $C$ be a self-dual code over $Z / 2$ and $W$ its complete weight enumerator polynomial. Then $W \in \mathbf{C}[f, g]$ where $f=x_{3}^{2}+x_{1}^{2}$ and $g=x_{0}^{2} x_{1}^{2}\left(x_{0}^{2}-x_{1}^{2}\right)^{2}$.

We now consider the case $p=3$ and take a self-dual linear code $C$ on $V \equiv V_{n}$ satisfying $1_{n} \in C$. It is well known that 12 divides $n$ and, hence, $W \equiv W_{C}$ lies in $A^{\prime \prime \prime}$. Gleason's theorem describes the structure of $A^{\prime \prime \prime}$. 
We begin by noting that the algebra homomorphism $\Psi^{*}: A^{\prime \prime \prime} \rightarrow \Theta^{\prime \prime \prime}$, is not a monomorphism. Let $K$ be the kernel of $\Psi^{*}$ on $A^{\prime \prime \prime}$. The description of $A^{\prime \prime \prime}$ will be of a more complicated nature than the corresponding description of $A^{\prime}$ in the $p=2$ case. From our approach the reason for this is the existence of the nontrivial kernel $K$.

We require the following notation. Let

$$
a \equiv x_{0}^{3}+x_{1}^{3}+x_{2}^{3}, \quad p \equiv 3 x_{0} x_{1} x_{2}, \quad b \equiv x_{0}^{3} x_{1}^{3}+x_{0}^{3} x_{2}^{3}+x_{1}^{3} x_{2}^{3},
$$

and

$$
\begin{aligned}
\alpha_{12} & =a\left(a^{3}+8 p^{3}\right), \quad \beta_{6}=a^{2}-12 b, \quad \nu_{18}=a^{6}-20 a^{3} p^{3}-8 p^{6}, \\
\delta_{36} & =p^{3}\left(a^{3}-p^{3}\right)=-\frac{1}{64}\left(\nu_{18}^{2}-\alpha_{12}^{3}\right) .
\end{aligned}
$$

A direct computation shows that, under the action of $F_{S}$, we have

$$
a \rightarrow \frac{1}{\sqrt{3}}(a+2 p), \quad p \rightarrow \frac{1}{\sqrt{3}}(a-p), \quad b \rightarrow-b+\frac{1}{9}\left(a^{2}+a p+p^{2}\right),
$$

from which we infer that the action of $F_{S}$ has the following effect:

$$
\begin{gathered}
\alpha_{12} \rightarrow \alpha, \quad \beta_{6}^{2} \rightarrow \beta_{6}^{2}, \quad \alpha_{36} \rightarrow \alpha_{36}, \\
\beta_{6} \rightarrow-\beta_{6}, \quad r_{18} \rightarrow-r_{18} .
\end{gathered}
$$

By (4.4) it follows that $\alpha_{12}, \beta_{6}^{2}, \alpha_{36}$ lie in $A^{\prime \prime \prime}$ and they can be shown to freely generate.

THEOREM 4.1. $A^{\prime \prime \prime}=\mathbf{C}\left[\alpha_{12}, \beta_{6}^{2}\right] \oplus K$, where $K$ is the kernel of $\Psi^{*}$ in $A^{\prime \prime \prime}$.

Proof. Clearly, $\Psi^{*}(a), \Psi^{*}(p)$ are elements in $\Theta_{1}$. Since $\Theta_{1}$ is one dimensional and $F_{L}$ acts by the identity mapping on $\Theta_{1}$, we have

$$
\Psi^{*}(p)=\mu \cdot \Psi^{*}(a), \quad \mu \in C, \quad F_{L}\left(\Psi^{*}(a)\right)=\Psi^{*}(a) .
$$

By (4.3) we determine $\mu=(-1+\sqrt{3}) / 2$. This implies $\Psi^{*}\left(\alpha_{12}\right)=\left(1+8 U^{2}\right) \Psi^{*}(a)^{4} \neq$ 0 , so $\Psi^{*}\left(\alpha_{12}\right) \in \Theta_{4} \cap \Theta^{\prime}$. Let $y_{1}=\Psi^{*}(a)^{2}, y_{2}=\Psi^{*}\left(\beta_{6}\right)$. Then $F_{L}\left(y_{1}\right)=$ $y_{1}, F_{L}\left(y_{2}\right)=-y_{2}$, which, by the Appendix, implies $y_{1}, y_{2}$ freely generate. Thus, $y_{1}^{2}, y_{2}^{2}$ are linearly independent in $\Theta_{4} \cap \Theta^{\prime}$ and, by Theorem 3.12, freely generate $\Theta^{\prime \prime \prime}$. This completes the proof of the theorem.

Consider a polynomial $f\left(x_{0}, x_{1}, x_{2}\right)$ on $A^{\prime \prime \prime}$. Since $f\left(x_{0}, x_{1}, x_{2}\right)$ is $R$ - and $F_{S^{-}}^{2}$ invariant, where $F_{S}^{2}$ is given by the permutation of variables $x_{0} \mapsto x_{0}, x_{1} \mapsto$ $x_{2}, x_{2} \mapsto x_{1}$, we have that $f\left(x_{0}, x_{1}, x_{2}\right)$ is a symmetric polynomial in $x_{0}, x_{1}, x_{2}$. In addition, $f\left(x_{0}, x_{1}, x_{2}\right)$ is $U$-invariant. The above remarks imply that $f$ is also invariant under the transformations $x_{j} \mapsto e^{2 \pi i / 3} x_{j}$ for each $j=0,1,2$. . Thus, $f\left(x_{0}, x_{1}, x_{2}\right)$ is a symmetric polynomial in $x_{0}^{3}, x_{1}^{3}, x_{2}^{3}$. The theory of elementary symmetric polynomials implies we can write $F\left(x_{0}, x_{1}, x_{2}\right)$ as a polynomial on $a, p^{3}, b$.

We let $f\left(x_{0}, x_{1}, x_{2}\right) \equiv f^{\prime}\left(a, p^{3}, b\right)$. The degree of each monomial $x^{r}=x_{0}^{r_{0}} x_{1}^{r_{1}} x_{2}^{r_{2}}$ appearing in $F\left(x_{0}, x_{1}, x_{2}\right)$ is divisible by 12 . This implies that a monomial $a^{s_{0}} p^{3 s_{1}} b^{s_{2}}$, which appears as a term in $f^{\prime}\left(a, p^{3}, b\right)$, must satisfy $4 / s_{0}+3 s_{1}+2 s_{2}$.

The algebra $A^{\prime \prime \prime}$ can be described as the space of all polynomials $f^{\prime}\left(a, p^{3}, b\right)$ in the linear span of monomials $a^{s_{0}} p^{3 s_{1}} b^{s_{2}}$ satisfying

$$
4 / s_{0}+3 s_{1}+2 s_{2}
$$


which are invariant under the transformations given by (4.3).

We want to describe $A^{\prime \prime \prime}$ and we know that $A^{\prime \prime \prime}=\mathbf{C}\left[\alpha_{12}, \beta_{6}^{2}\right] \oplus K$, where $K$ is the kernel of $\Psi^{*}$ in $A^{\prime \prime \prime}$. Hence, we begin by giving a characterization of $K$.

Let $K^{\prime}$ be the collection of all polynomials $f^{\prime}\left(a, p^{3}, b\right) \in \mathbf{C}\left[a, p^{3}, b\right]$ such that

$$
\gamma_{18} \cdot f\left(a, p^{3}, b\right) \in A^{\prime \prime \prime} \text {. }
$$

In particular, we observe that because $F_{S}\left(\gamma_{18}\right)=-\gamma_{18}$, we have $F_{S}\left(f\left(a, p^{3}, b\right)\right)=$ $-f\left(a, p^{3}, b\right)$.

THEOREM 4.2. $K=\gamma_{18} \cdot K^{\prime}$.

PROOF. From $\gamma_{18}=a^{6}-20 a^{3} p^{3}-8 p^{6}$, it follows that

$$
\psi^{*}\left(\gamma_{18}\right)=\left(1-20 u^{3}-8 u^{6}\right) \Psi^{*}(a),
$$

where $u=(-1+\sqrt{3}) / 2$. A direct calculation shows that $1-20 u^{3}-8 u^{6}=0$. This implies $\gamma_{18} K^{\prime} \subset K$.

Consider $k\left(a, p^{3}, b\right) K$ and let $k^{\prime}(a, b)=k\left(a, u^{3} a^{3}, b\right)$. Then

$$
k^{\prime}\left(\Psi^{*}(a), \Psi^{*}(b)\right)=k\left(\Psi^{*}(a), \Psi^{*}\left(p^{3}\right), \Psi^{*}(b)\right)=0,
$$

which implies, since $\Psi^{*}(a), \Psi^{*}(b)$ are freely generating, that $k^{\prime}(a, b)=0$. Thus, $(u a-p) \mid k\left(a, p^{3}, b\right)$ in $\mathbf{C}[a, p, b]$.

Since $k\left(a, p^{3}, b\right)$ is invariant under the action, $p \rightarrow w p, w=\exp (2 \pi i / 3)$, it follows that $u a-w p, u a-w^{2} p$ are factors of $k\left(a, p^{3}, b\right)$. Thus, since $u a-p, u a-w p, u a-w^{2} p$ are relatively prime, we have $u^{3} a^{3}-p^{3}=(u a-p)(u a-w p)\left(u a-w^{2} p\right)$ is a factor of $k\left(a, p^{3}, b\right)$.

Consider the factor $u a-w p$. Since $k\left(a, p^{3}, b\right)$ is invariant under $F_{S}$, we have $F_{S}(u a-w p)$ is a factor of $k\left(a, p^{3}, b\right)$. This implies, by a direct calculation, that $v a-p, v=(-1-\sqrt{3}) / 2$, is a factor of $k\left(a, p^{3}, b\right)$. Arguing as above, we have

$$
\gamma_{18}=(-8)\left(u^{3} a^{3}-p^{3}\right)\left(v^{3} a^{3}-p^{3}\right)
$$

is a factor of $k\left(a, p^{3}, b\right)$. This completes the proof of the theorem.

The final stage in the proof of Gleason's theorem when $p=3$ is to describe $K^{\prime}$. We quickly outline how this is done. For $f\left(a, p^{3}, b\right) \in K^{\prime}$ we have $F_{S}(f)=-f$. Also, each monomial in $f\left(a, p^{3}, b\right), a^{s_{0}} p^{3 s_{1}} b^{s_{2}}$, has the property that $\left(s_{0}+3 s_{1}+2 s_{2}\right) / 2$ is odd.

Arguing as in the remarks leading to the description of $A^{\prime \prime \prime}$ in Theorem 4.1, we can prove

THEOREM 4.3. If $f\left(a, p^{3}, b\right) \in K^{\prime}$ then

$$
f\left(a, p^{3}, b\right)=\beta_{6} g\left(\alpha_{12}, \beta_{6}^{2}\right)+\gamma_{18} f^{\prime}\left(a, p^{3}, b\right),
$$

where $f^{\prime}\left(a, p^{3}, b\right) \in A^{\prime \prime \prime}$.

We come now to the main theorem in the case $p=3$.

THEOREM D. $A^{\prime \prime \prime}=\mathbf{C}\left[\alpha_{12}, \beta_{6}^{2}, \delta_{36}\right] \oplus \beta_{6} \gamma_{18} \mathbf{C}\left[\alpha_{12}, \beta_{6}^{2}, \delta_{36}\right]$, where $\alpha_{12}, \beta_{6}^{2}, \delta_{36}$ freely generate.

PROOF. Assume $\alpha_{12}, \beta_{6}^{2}, \delta_{36}$ freely generate. Also, the sum on the right is trivially a direct sum contained in $A^{\prime \prime \prime}$. 
Consider $f\left(a, p^{3}, b\right) \in A^{\prime \prime \prime}$. Without loss of generality we assume that $f\left(a, p^{3}, b\right)$ is homogeneous in $x_{0}, x_{1}, x_{2}$ of degree $m$. Thus, each monomial $a^{s_{0}} p^{3 s_{1}} b^{s_{2}}$ occurring in $f\left(a, p^{3}, b\right)$ satisfies $m=3 s_{0}+9 s_{1}+6 s_{2}$. We use induction on $m$.

By Theorem 4.1 we can write

$$
f\left(a, p^{3}, b\right)=g\left(\alpha_{12}, \beta_{6}^{2}\right)+k\left(a, p^{3}, b\right),
$$

where $k\left(a, p^{3}, b\right) \in K$. Applying Theorem 4.2 we can write

$$
k\left(a, p^{3}, b\right)=\gamma_{18} k^{\prime}\left(a, p^{3}, b\right),
$$

where $k^{\prime}\left(a, p^{3}, b\right) \in K^{\prime}$. Theorem 4.3 implies

$$
k^{\prime}\left(a, p^{3}, b\right)=\beta_{6} g^{\prime}\left(\alpha_{12}, \beta_{6}^{2}\right)+\gamma_{18} f^{\prime}\left(a, p^{3}, b\right),
$$

where $f^{\prime}\left(a, p^{3}, b\right) \in A^{\prime \prime \prime}$. The degree of $f^{\prime}$ as a polynomial in $x_{0}, x_{1}, x_{2}$ is strictly less than $m$, which implies by induction that

$$
f^{\prime}\left(a, p^{3}, b\right) \in \mathbf{C}\left[\alpha_{12}, \beta_{6}^{2}, \delta_{36}\right] \oplus \beta_{6} \gamma_{18} \mathbf{C}\left[\alpha_{12}, \beta_{6}^{2}, \delta_{36}\right] .
$$

Putting all this together we get

$$
f\left(a, p^{3}, b\right)=g\left(\alpha_{12}, \beta_{6}^{2}\right)+\gamma_{18}^{2} f^{\prime}\left(a, p^{3}, b\right)+\gamma_{18} \beta_{6} g^{\prime}\left(\alpha_{12}, \beta_{6}^{2}\right) .
$$

Since $\gamma_{18}^{2}=-64 \delta_{36}+\alpha_{12}^{3}$, the theorem is proved.

GLEASON'S THEOREM FOR $p=3$. Let $C$ be a self-dual code over $Z / 3$ and $W$ its weight enumerator polynomial. Suppose $1 \in C$. Then

$$
W \in \mathbf{C}\left[\alpha_{12}, \beta_{6}^{2}, \delta_{36}\right] \oplus \beta_{6} \gamma_{18} \mathbf{C}\left[\alpha_{12}, \beta_{6}^{2}, \delta_{36}\right] .
$$

Appendix. The structure of the algebra $L^{*}$, especially the relationship of this structure to the action of the algebra homomorphism $F_{L}$, is the central tool in the second part of this work. In this Appendix we review the results we need.

We begin with the direct sum decomposition for $L^{*}, L^{*}=\sum_{m \geq 0} \bigoplus L_{m}$, and the elements $z_{1} \in L_{1}, z_{2} \in L_{2}, z_{3} \in L_{3}$, where

$$
F_{L}\left(z_{1}\right)=z_{1}, \quad F_{L}\left(z_{2}\right)=-z_{2}, \quad F_{L}\left(z_{3}\right)=i z_{3},
$$

and $L^{*}=\mathbf{C}\left[z_{1}, z_{2}, z_{3}\right]$. The elements $z_{1}, z_{2}, z_{3}$ do not freely generate.

Consider the action of $F_{L}$ on $L^{*}$. Denote the subspace of $L^{*}$ spanned by the

\begin{tabular}{|c|c|c|}
\hline Subspace & \multicolumn{2}{|c|}{ Basis } \\
\hline & +1 & -1 \\
\hline$M_{1}$ & $z_{1}$ & \\
\hline$M_{2}$ & $z_{1}^{2}$ & $z_{2}$ \\
\hline$M_{3}$ & $z_{1}^{3}$ & $z_{1} z_{2}$ \\
\hline$M_{4}$ & $z_{1}^{4}, z_{2}^{2}$ & $z_{1}^{2} z_{2}$ \\
\hline$M_{5}$ & $z_{1}^{5}, z_{1} z_{2}^{2}$ & $z_{1}^{3} z_{2}$ \\
\hline$M_{6}$ & $z_{1}^{6}, z_{1}^{2} z_{2}^{2}$ & $z_{1}^{4} z_{2}, z_{2}^{3}$ \\
\hline$M_{7}$ & $z_{1}^{7}, z_{1}^{3} z_{2}^{2}$ & $z_{1}^{5} z_{2}, z_{1} z_{2}^{3}$ \\
\hline$M_{8}$ & $z_{1}^{8}, z_{1}^{4} z_{2}^{2}, z_{2}^{4}$ & $z_{1}^{6} z_{2}, z_{1}^{2} z_{2}^{3}$ \\
\hline
\end{tabular}
eigenvectors of $F_{L}$ having eigenvalues \pm 1 by $M$ and the eigenvalue one subspace of $F_{L}$ on $L^{*}$ by $M^{\prime}$. Then $M$ is a subalgebra of $L^{*}$, and $M^{\prime}$ is a subalgebra of $M$. In fact, we have $M=M^{\prime} \oplus z_{2} M^{\prime}$, where $M^{\prime}$ is generated freely by $z_{1}, z_{2}^{2}$, and $M$ is generated freely by $z_{1}, z_{2}$.

These results, along with those mentioned below, are immediate consequences of the following tables. Let $M_{n} \equiv L_{m} \cap M, M_{m}^{\prime} \equiv L_{m} \cap M^{\prime}$. 
In general, we distinguish four cases for $M_{m}$ :

$$
\begin{array}{ll}
\multicolumn{1}{c}{+} & - \\
z_{1}^{m}, z_{1}^{m-4} z_{2}^{2}, \ldots, z_{2}^{m / 2} & z_{1}^{m-2} z_{2}, z_{1}^{m-6} z_{2}^{3}, \ldots, z_{1}^{2} z_{2}^{(m-2) / 2} \\
m \equiv 1 \bmod 4 & \\
z_{1}^{m}, z_{1}^{m-4} z_{2}^{2}, \ldots, z_{1} z_{2}^{(m-1) / 2} & z_{1}^{m-2} z_{2}, z_{1}^{m-6} z_{2}^{3}, \ldots, z_{1}^{3} z_{2}^{(m-3) / 2} \\
m \equiv 2 \bmod 4 & \\
z_{1}^{m}, z_{1}^{m-4} z_{2}^{2}, \ldots, z_{1}^{2} z_{2}^{(m-2) / 2} & z_{1}^{m-2} z_{2}, z_{1}^{m-6} z_{2}^{3}, \ldots, z_{2}^{m / 2} \\
m \equiv 3 \bmod 4 & \\
z_{1}^{m}, z_{1}^{m-4} z_{2}^{2}, \ldots, z_{1}^{3} z_{2}^{(m-3) / 4} & z_{1}^{m-2} z_{2}, z_{1}^{m-6} z_{2}^{3}, \ldots, z_{1} z_{2}^{(m-1) / 2}
\end{array}
$$

From the pattern established by these tables, statements (1)-(3) easily follow.

(1) $M \cap \sum_{m \geq 0} \bigoplus L_{2 m}$ is generated by $z_{1}^{2}, z_{2}$.

(2) $M^{\prime} \cap \sum_{m \geq 0} \bigoplus L_{2 m}$ is generated by $z_{1}^{2}, z_{2}^{2}$.

(3) $M^{\prime} \cap \sum_{m \geq 0} \bigoplus L_{4 m}$ is generated by $z_{1}^{4}, z_{2}^{2}$.

We consider generalizations of these results, which are continually used in this work. Although more general results can be appealed to, the grading in $L^{*}$, i.e., the direct sum decomposition of $L^{*}$, makes the proofs especially simple.

Let $y_{2} \in M_{2}$ and $y_{4}, y_{4}^{\prime} \in M_{4}^{\prime}$ in the following statements:

(1) If $z_{1}^{2}, y_{2}$ are linearly independent, then $z_{1}, y_{2}$ freely generate $M$.

(2) If $z_{1}^{4}, y_{4}$ are linearly independent, then $z_{1}, y_{4}$ freely generate $M^{\prime}$.

(3) If $y_{2} \in M^{\prime}$ and $y_{2}^{2}, y_{4}$ are linearly independent, then $y_{2}, y_{4}$ freely generate $M^{\prime} \cap \sum_{m \geq 0} \bigoplus L_{2 m}$.

(4) If $y_{4}, y_{4}^{\prime}$ are linearly independent, they freely generate $M^{\prime} \cap \sum_{m>0} \bigoplus L_{4 m}$.

We prove only the first statement. The others follow in much the same way. Write $y_{2}=c z_{1}^{2}+d z_{2}$, where, by assumption, $d \neq 0$. $M$ is generated freely by $z_{1}, z_{2}$, and since every monomial $z_{1}^{a_{1}} z_{2}^{a_{2}}$ can be written $z_{1}^{a_{1}} z_{2}^{a_{2}}=z_{1}^{a_{1}}\left[\left(y_{2}-c z_{1}^{2}\right) d^{-1}\right]^{a_{2}}$ upon expanding, we have $M$ generated by $z_{1}, y_{2}$.

Consider any polynomial $f\left(z_{1}, y_{2}\right)$ satisfying $f\left(z_{1}, y_{2}\right)=0$. Write

$$
f\left(z_{1}, y_{2}\right)=\sum_{r=0}^{N} f_{r}\left(z_{1}\right) y_{2}^{r}
$$

where we assume $f_{N}\left(z_{1}\right) \neq 0$. Replace $y_{2}$ by $c z_{1}^{2}+d z_{2}$ in this expression and form

$$
g\left(z_{1}, z_{2}\right)=f\left(z_{1}, c z_{1}^{2}+d z_{2}\right)=\sum_{r=0}^{N} f_{r}\left(z_{1}\right)\left(c z_{1}^{2}+d z_{2}\right)^{r}
$$

The highest power of $z_{2}$ which occurs upon expanding is $z_{2}^{N}$ and its coefficient is $f_{N}\left(z_{1}\right) d^{N}$. Since $d \neq 0, z_{1}, z_{2}$ freely generate, and $g\left(z_{1}, z_{2}\right)=0$, we must have $f_{N}\left(z_{1}\right)=0$, a contradiction. Thus, $z_{1}, y_{2}$ freely generate. 


\section{REFERENCES}

1. L. Auslander and R. Tolimieri, Algebraic structures for $\bigoplus \sum_{n>1} L^{2}(Z / n)$ compatible with the finite Fourier transform, Trans. Amer. Math. Soc. 244 (1978), 263-272.

2. Is computing with the finite Fourier transform pure or applied mathematics, Bull. Amer. Math. Soc. (N.S.) 1 (1979), 847-897.

3. A. Gleason, Weight polymomials of self-dual codes and the MacWilliams identities, Actes Congrès Internat. Math., Vol. 3, Gauthier-Villars, Paris, 1971, pp. 211-215.

4. F. J. MacWilliams, $A$ theorem on the distribution of weights in a systematic code, Bell System Tech. J. 42 (1963), 79-84.

5. F. J. MacWilliams, C. Mallows and N. Sloane, Generalizations of Gleason's theorem on weight enumerators of self-dual codes, IEEE Trans. Inform. Theory 18 (1972), 794-805.

6. F. MacWilliams and N. Sloane, The theory of error correcting codes, North-Holland, Amsterdam, 1978.

7. J. P. Serre, A course in arithmetic, Graduate Texts in Math., Springer-Verlag, New York.

8. N. J. Sloane, Weight enumerators of codes, Combinatorics (M. Hall, Jr. and J. H. van Lont, eds.), Reidel, Dordrecht, 1975, pp. 115-142.

9. $\ldots$, Error-correcting codes and invariant theory, Amer. Math. Monthly 84 (1977), 82-107.

10. R. E. Blahut, Theory and practice of error control codes, Addison-Wesley, Reading, Mass., 1983.

Department of Electrical Engineering, City COllege of NeW York, CUNY, NEW YORK, NEW YORK 10031 